\title{
Positional frequency and versatility of letters for six-, seven-, and eight-letter English words
}

\author{
ROBERT L. SOLSO \\ University of Idaho, Moscow, Idaho 83843
}

\begin{abstract}
The positional frequency and versatility of letters were tabulated for six-, seven-, and eight-letter English words.
\end{abstract}

As the interest in language processing has increased, so too has the need for more sensitive statistical descriptions. Gross counts of letter and letter combination frequencies (e.g., Underwood \& Schulz, 1960) gave way to letter and letter combination frequencies based on the position each letter occupied in a word (Mayzner \& Tresselt, 1965). Another descriptive statistic, versatility, in which letters and letter combinations are evaluated in terms of the number of different words in which they appear, was introduced by Solso and King (1976) and by Topper, Macey, and Solso (1973).

The previously reported frequency and versatility counts by Solso and King (1976) were limited to positional statistics based on four- and five-letter words.
Current research of letter and word identification has demanded accurate positional statistics of longer words. The present paper provides research with positional frequency and versatility of six-, seven-, and eight-letter words.

\section{METHOD}

Positional frequency and versatility counts for six-, seven-, and eight-letter words were based on the Kučera and Francis (1967) frequency count of about 1 million English words. In the present analysis, all hyphenated words, words containing apostrophes, and numbers were excluded.

Frequency counts by position were based on the total times a letter appeared in each position; versatility counts were based on the total number of times a letter appeared in a different

Table 1

Letter Frequency and Versatility by Position for Six-Letter Words

\begin{tabular}{|c|c|c|c|c|c|c|c|c|c|c|c|c|c|c|}
\hline & \multicolumn{12}{|c|}{ Position } & & \\
\hline & \multicolumn{2}{|c|}{1} & \multicolumn{2}{|c|}{2} & \multicolumn{2}{|c|}{3} & \multicolumn{2}{|c|}{4} & \multicolumn{2}{|c|}{5} & \multicolumn{2}{|c|}{6} & \multicolumn{2}{|c|}{ Total } \\
\hline & F & V & F & V & F & V & F & V & $\mathrm{F}$ & V & $\mathbf{F}$ & V & $\mathrm{F}$ & V \\
\hline A & 5869 & 303 & 12286 & 1019 & 6729 & 540 & 4586 & 377 & 3755 & 347 & 702 & 183 & 33927 & 2769 \\
\hline B & 5690 & 444 & 300 & 25 & 1296 & 14 & 1465 & 14 & 114 & & 50 & 11 & 8915 & 796 \\
\hline C & 6726 & 436 & 1839 & 68 & 4000 & 212 & 1654 & 148 & 3549 & 164 & 800 & 54 & 18568 & 1082 \\
\hline D & 3460 & 287 & 359 & 43 & 1947 & 186 & $30 \mathrm{c}$ & 26 & 1428 & 125 & 14047 & 760 & 24243 & 1669 \\
\hline E & 3455 & 173 & 15327 & 839 & 3954 & 334 & 8114 & 504 & 25134 & 1821 & 13443 & 694 & 69427 & 4365 \\
\hline F & 4260 & 272 & 915 & 23 & 2515 & 85 & 449 & 77 & 103 & 38 & 603 & 16 & 8945 & 411 \\
\hline G & 2084 & 270 & 23 & 18 & 195 & 161 & 2745 & 209 & 2500 & 79 & 3900 & 175 & 13418 & 912 \\
\hline $\mathrm{H}$ & 2632 & 246 & 4764 & 240 & 135 & 64 & 2937 & 13 & 1178 & 89 & 2656 & 118 & 15520 & 894 \\
\hline I & 1764 & 99 & 8058 & 571 & 478 & 383 & 11384 & 65 & 3546 & 383 & 112 & 42 & 29644 & 2129 \\
\hline J & & 84 & 6 & 2 & 155 & 18 & 6 & 3 & 0 & 0 & 0 & 0 & 803 & 107 \\
\hline $\mathrm{K}$ & 35 & 78 & 111 & 17 & 775 & 39 & 2227 & 203 & 655 & 96 & 270 & 3 & 43 & 470 \\
\hline L & 4259 & 259 & 3340 & 282 & 5012 & 438 & 3944 & 39 & 9111 & 432 & 2977 & 209 & 28643 & 2018 \\
\hline M & 5156 & 314 & 581 & 45 & 4121 & 220 & 2396 & 20 & 1382 & 85 & 1137 & 88 & & 957 \\
\hline $\mathrm{N}$ & 2175 & 117 & & 213 & 5042 & 448 & 4837 & 347 & 8488 & 444 & 6228 & 517 & 30281 & 2086 \\
\hline 0 & 1502 & 109 & 12068 & 821 & 7126 & 355 & 6265 & 302 & 5753 & 350 & 358 & 92 & & 2029 \\
\hline $\mathbf{P}$ & 6248 & 378 & 1246 & 94 & 1572 & 151 & 2570 & 170 & 878 & 59 & 144 & 28 & 12658 & 980 \\
\hline $\mathrm{Q}$ & 119 & 22 & 191 & 16 & 94 & 5 & 99 & 9 & 0 & 0 & & & 503 & 52 \\
\hline $\mathrm{R}$ & 4607 & 317 & 5450 & 463 & 9920 & 628 & 4104 & 302 & 6522 & 348 & 8837 & 553 & 39440 & 2611 \\
\hline $\mathrm{s}$ & 12309 & 708 & 897 & 53 & 5059 & 342 & 3161 & 272 & 2983 & 204 & 12062 & 109 & & 2678 \\
\hline$T$ & 4309 & 315 & & 152 & 7903 & 325 & 8258 & 455 & 3462 & 294 & 7359 & 370 & 34878 & 1911 \\
\hline $\mathrm{U}$ & 1152 & 81 & 5898 & 496 & 3076 & 232 & 6121 & 186 & 1307 & 165 & 84 & 16 & 17638 & 1176 \\
\hline V & 1198 & 108 & 200 & 20 & 1711 & 118 & 1151 & 125 & 390 & 31 & 3 & 3 & 4653 & 405 \\
\hline W & 2822 & 189 & 333 & 39 & 1458 & 82 & 841 & 66 & 127 & 24 & 723 & 47 & 6304 & 447 \\
\hline $\mathrm{X}$ & & 1 & 731 & 34 & 199 & 27 & 26 & 10 & 5 & 2 & 82 & 23 & 1044 & 97 \\
\hline $\mathrm{Y}$ & 170 & 27 & 737 & 53 & 1106 & 81 & 428 & 34 & 533 & 31 & 6373 & 503 & 9347 & 729 \\
\hline \multirow[t]{2}{*}{$\mathrm{Z}$} & 26 & 14 & 12 & $=$ & 119 & 30 & 209 & 51 & 76 & 13 & 29 & 13 & 471 & 126 \\
\hline & 82979 & 5651 & 82979 & 5651 & 82979 & 5651 & 82979 & 5651 & 82979 & 5651 & 82979 & 5651 & 497974 & \\
\hline
\end{tabular}




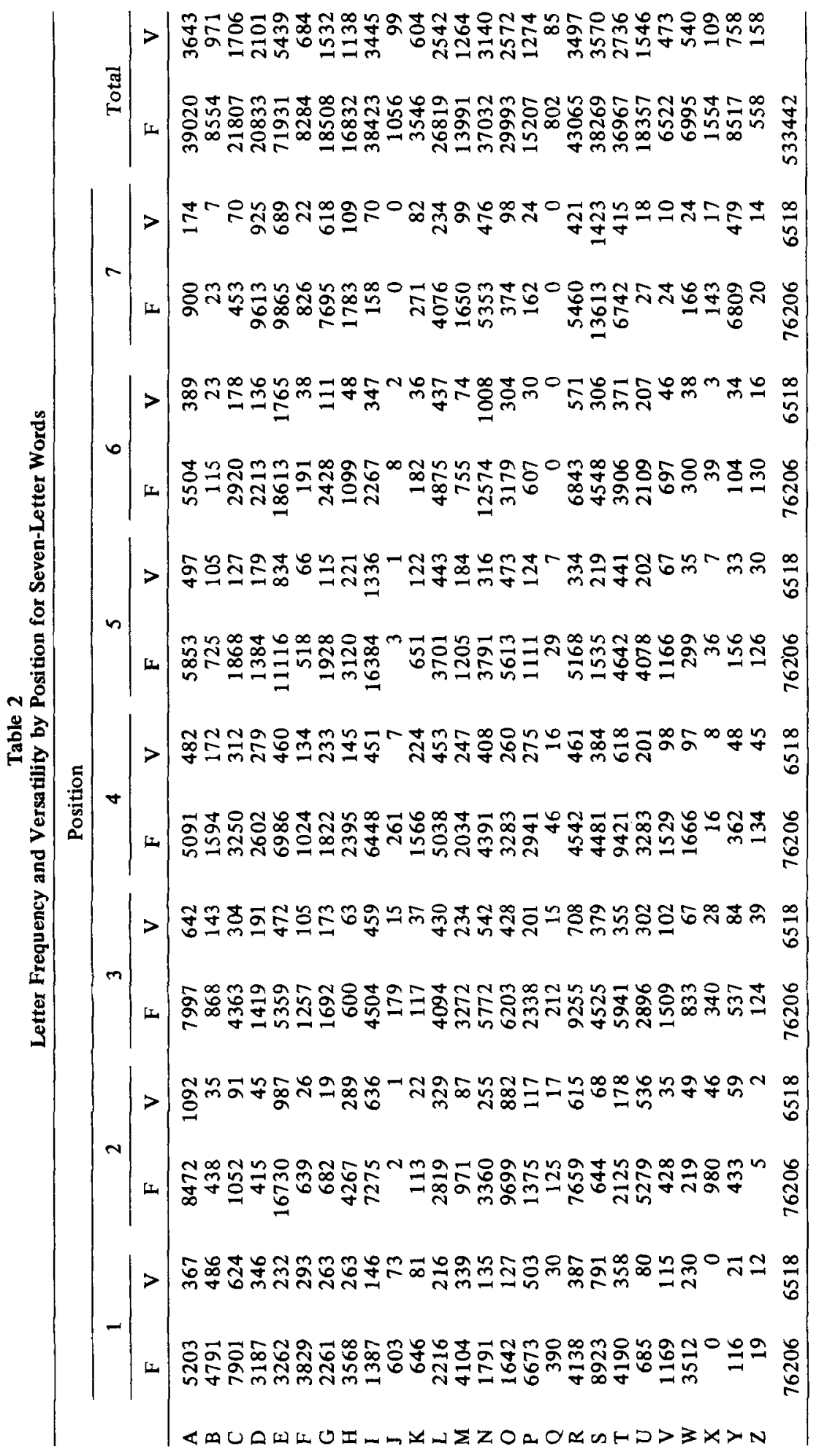




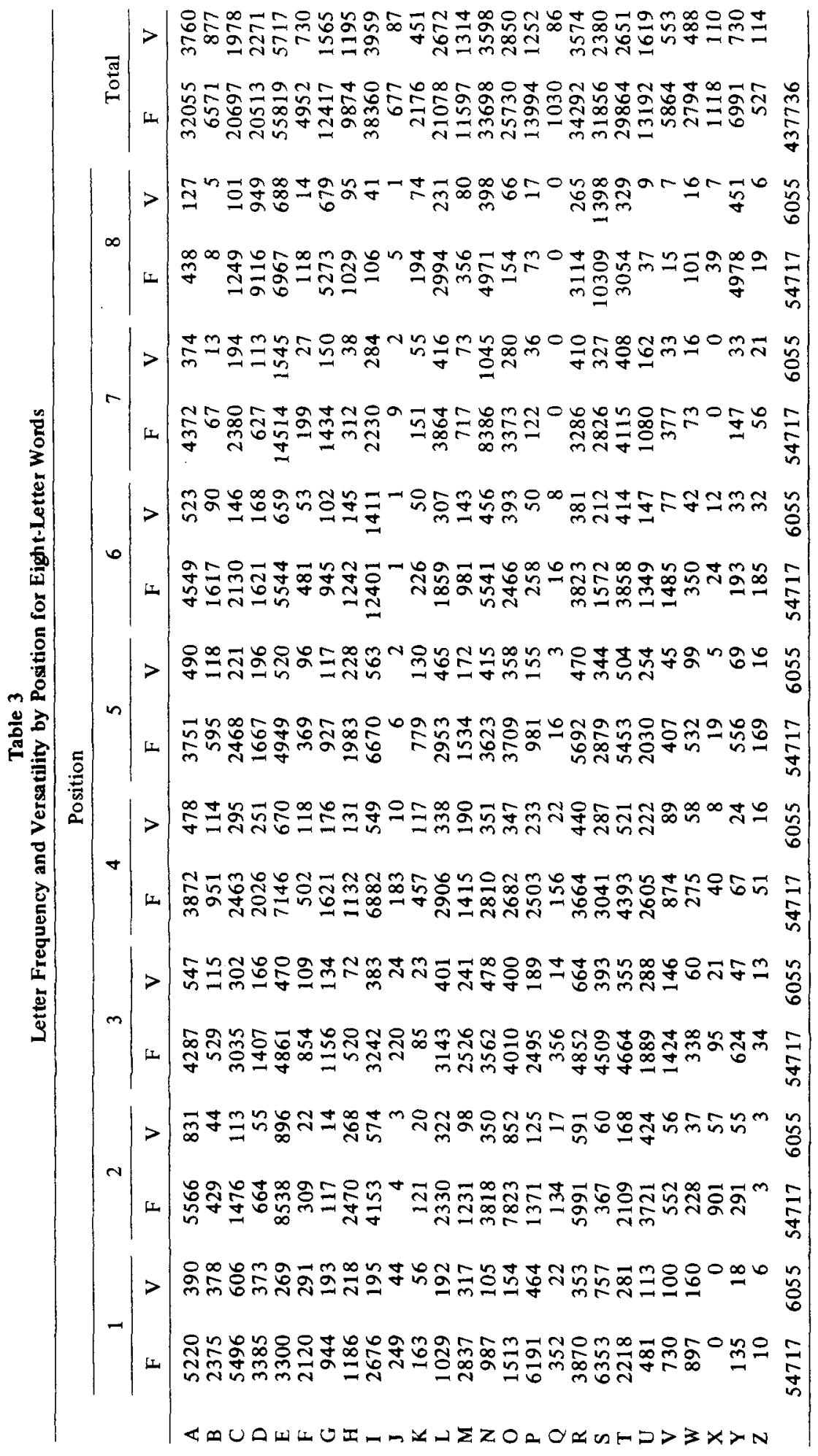


word. For example, the frequency count for the word "junior" has a total frequency of 75 per million words. The frequency totals by position for the letters $J, U, N, I, O$, and $R$ were incremented by 75 ; for the versatility count by position, each letter was incremented by 1 . The word "attack" has a frequency of 105 million: "A," which appears in the first and fourth positions, was incremented by 105 for the first position and 105 for the fourth position. The letter $T$ was similarly treated for the second and third positions, while $C$ and $K$ were incremented by 105 for their respective positions. The versatility by position of each letter in the word was incremented by 1 .

\section{RESULTS}

Total frequency and versatility counts for six-, seven-, and eight-letter words are contained in Tables 1, 2, and 3 .

\section{REFERENCES}

Kučera, H., \& Francis, W. N. Computational analysis of present-day American English. Providence, R.I: Brown University Press, 1967.

Mayzner, M. S., \& Tresselt, M. E. Table of single-letter and bigram frequency counts for various word-length and letterposition combinations. Psychonomic Monograph Supplements, 1965, 1(Whole No. 2).

Solso, R. L., \& King, J. F. Frequency and versatility of letters in the English language. Behavior Research Methods \& Instrumentation, 1976, 8, 283-285.

Topper, G. E., Macey, W. H., \& Solso, R. L. Bigram versatility and bigram frequency. Behavior Research Methods \& Instrumentation, 1973, 5, 51-53.

UNDE RWOOD, B. J., \& Schulz, R. W. Meaningfulness and verbal learning. Chicago: Lippencott, 1960.

(Received for publication January 26, 1979; revision accepted March 30, 1979.) 